\title{
ÍNDICE DE EROSIÓN-SEDIMENTACIÓN COSTERA (IE-SC): UNA APLICACIÓN EN LA COSTA DEL PACÍFICO CENTRAL DE COSTA RICA
}

\author{
Guaria Cárdenes \& Luis Gmo. Obando \\ Escuela Centroamericana de Geología, Apdo. 214-2060 Universidad de Costa Rica. \\ E-mail:gcardene@geologia.ucr.ac.cr \\ E-mail: lobando@geologia.ucr.ac.cr
}

(Recibido 6/9/05; Aceptado 14/2/06)

\begin{abstract}
A semi-quantitative methodology index has been designed. The Coastal Erosion-Sedimentation Index (IE-SC) is designed for the study of intense erosion-sedimentation coastal processes that can affect the human activities.

The IE-SC index is based on three different kind of evidences, a: on the flora, b: on the infrastructure, and c: at the physical geology environment (unconsolidated materials). The parameters used during the evaluation are, among others, erosion terraces formation, roots exposure within/or close to the foreshore zone, soft and hard coastal protection infrastructure presence. Maps were constructed in order to show the analyzed data; different levels or weights, ranging 1 to 5, were assigned to the different coastal areas. Level 1 zones indicates that intense erosion-sedimentation processes that can affect human activities are not present. Levels 2 and 3 present light evidence of erosion-sedimentation processes. Levels 4 and 5 correspond with zones that are highly affected by erosion-sedimentation processes, including damage and/or complete infrastructure loss. This scale was established in order to determine coastal zones that present intense erosion-sedimentation problems for human activities. It is expected that this methodology can help with coastal management programs.

As examples, Two Costa Rican Central Pacific areas are shown, where the IE-SC index has been implemented. The first one is located in Damas, Quepos and the other corresponds with the Tárcoles river mouth. At both zones intense erosive process predominate over the sedimentation, causing even total infrastructure loss. Sedimentation processes are also intense and are, mainly, the eroded materials resedimentation. These two areas present mostly, 4 and 5 IE-SC index levels.
\end{abstract}

Keywords: Central pacific coastal line, Damas Island sand bar, Tárcoles river, erosion-sedimentation index, coastal processes, coastal management.

RESUMEN: Se diseñó una metodología de tipo semi-cuantitativa llamada Índice de Erosión-Sedimentación Costera (IE-SC), para el estudio de los intensos procesos de erosión-sedimentación en áreas costeras que podrían afectar las actividades humanas. Las evidencias de erosión-sedimentación utilizadas en esta metodología son de tres tipos a: evidencias en la vegetación, b: evidencias en la infraestructura, y c: evidencias en el medio físico-geológico (materiales inconsolidados). Los parámetros que se utilizan para realizar la evaluación de la costa son, entre otros, formación de terrazas de erosión, exposición de raíces de árboles cercanos a la zona intermareal o bien dentro de ella, presencia de infraestructura que tiene como objetivo la "estabilización" y/o contención de erosión-sedimentación, etc. Los datos del análisis se presentan por medio de mapas donde a las distintas áreas costeras se les asigna un nivel o peso entre 1 y 5 . Zonas con peso 1 son aquellas que no presentan hasta el momento alguna evidencia de erosión-sedimentación intensa que podría afectar las actividades humanas, zonas clasificadas entre 2 y 3 presentan indicios de procesos erosivos y sedimentación, y áreas con pesos 4 y 5 
son zonas con considerables evidencias de erosión-sedimentación donde la infraestructura ya ha sido afectada o destruida por completo. Esta escala se ha establecido con el fin de determinar las zonas costeras que presentan problemas debido a erosión-sedimentación intensa, se espera que estos datos sean un insumo más para la elaboración de los planes de manejo de las áreas costeras. El IE-SC es una metodología nueva y se aplica por primera vez en Costa Rica.

Se muestran dos áreas en el Pacífico central costarricense, donde se ha implementado el IE-SC. La primera es la zona de Damas y la otra corresponde con la desembocadura del río Tárcoles. Ambas zonas se caracterizan por presentar un predominio de los procesos erosivos con respecto a la sedimentación, causando inclusive la pérdida total de infraestructura. Los procesos de sedimentación también son intensos, y constituyen, en su gran mayoría, la resedimentación de los materiales erosionados. En estas dos áreas se presentan niveles IE-SC entre 4 y 5.

Palabras clave: Costa del Pacífico central, barra arenosa de Damas, río Tárcoles, Índice de erosión-sedimentación, procesos costeros, manejo costero.

\section{INTRODUCCIÓN}

Debido al ascenso del nivel del mar, documentado a lo largo de vastas zonas costeras del planeta (p.ej. Anderson et al., 2001; Ortiz, 1994; Nichols, 1998), y al creciente desarrollo humano en las áreas costeras, se hace necesario el planteamiento de métodos tanto cualitativos como cuantitativos para determinar los posibles efectos de dicho ascenso en zonas costeras formadas por materiales sedimentarios inconsolidados. Se debe hacer énfasis en costas formadas por materiales inconsolidados ya que son las que pueden experimentar cambios morfológicos más drásticos y evidentes en menor tiempo, lo cual implica mayor afectación de las actividades humanas que se desarrollan en estas áreas.

Existen metodologías que evalúan cuantitativamente la vulnerabilidad costera, por ejemplo Gornitz et al. (1997), Thieler et al. (1999), Sharples (2004), Vafeidisa et al. (2004). Dichas propuestas se basan en el cálculo del índice de vulnerabilidad costera ante el ascenso del nivel del mar, el cual involucra una base de datos que relaciona parámetros tales como mareas, oleaje, evolución de la morfología de la costera, geomorfología actual, entre otros. Estas metodologías tienen la ventaja de tener una buena salida de datos, sin embargo su desarrollo puede ser muy costoso, lo hace que sea difícil de implementar en países de América Latina.

Dado que en Costa Rica no existen datos medidos de oleaje, entre otros parámetros que permitan determinar la vulnerabilidad de la zona costera ante los procesos de ascenso del nivel del mar por los métodos antes mencionados (incluyéndose en esta la vulnerabilidad del medio físico, de las actividades humanas y sobre todo de la infraestructura en la línea de costa), se ha diseñado una metodología que permita establecer zonas con patrones de erosión-sedimentación que podrían traer consecuencias negativas a las actividades humanas que se desarrollan en una costa particular. El Indice de erosión-sedimentación costera (IE-SC) se basa en la identificación y documentación de áreas donde prevalezca ya sea la erosión o la sedimentación, así como en la presencia de sistemas de estabilización costera (cualquier tipo de infraestructura civil orientada a la estabilización de la línea costera). Los sistemas de estabilización fueron tomados en cuenta a la hora de proponer la metodología ya que estos pueden influir positiva o negativamente en la contención de erosión-sedimentación, o sea, resultan ser elementos impuestos por los seres humanos que pasan a ser parte de la costa y que cambian su dinámica.

Por lo anterior, se diseñó un sistema de clasificación de tipo semi-cuantitativo para las áreas costeras conformadas por materiales inconsolidados ante procesos de erosión-sedimentación intensivos, los cuales pueden tener consecuencias negativas sobre las actividades humanas que se desarrollen. El método no contempla los procesos de erosión-sedimentación a lo largo de costas rocosas. Esta clasificación se ha establecido con el fin de determinar cuales zonas costeras y en que medida presentan fuertes cambios en los procesos de erosión-sedimentación, los cuales podrían causar desde pequeñas incomodidades hasta fuertes perdidas económicas en áreas costeras. La metodología está orientada a estudios 
diagnóstico de los eventos de erosión-sedimentación costera, donde esta esté conformada por materiales inconsolidados. Dicha metodología tiene como fin el ayudar a los planificadores (municipalidades, MINAE) de áreas costeras a establecer la pertinencia o no de desarrollar actividades específicas.

El área de estudio donde se diseñó y aplicó dicha metodología corresponde con la costa del Pacífico central de Costa Rica (Fig. 1). Se escogió dicha zona por dos razones, la primera debido a que se han documentado cambios importantes desde el punto de vista morfológico (Cárdenes, 2003; Lizano \& Salas, 2001; Lizano, 1997), y el otro factor que influyó fue el fácil acceso al área.

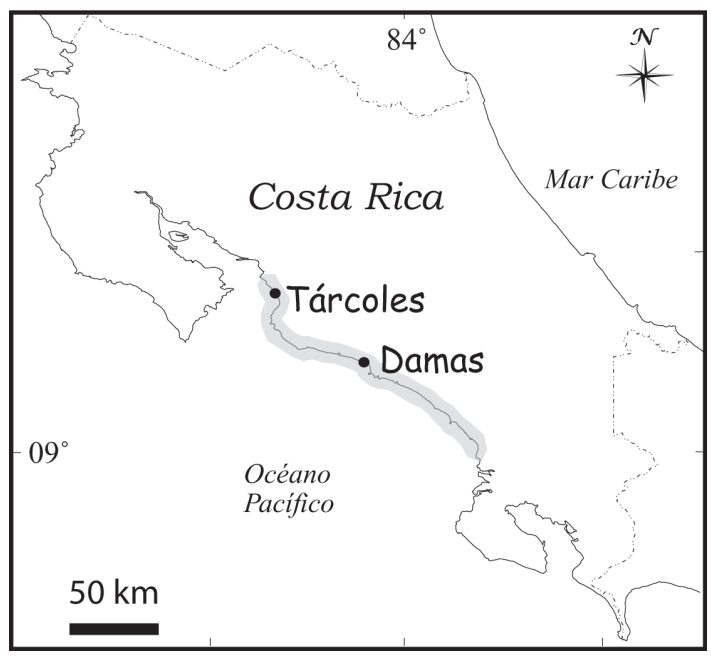

Fig.1: Ubicación de la zona de estudio. La zona costera del Pacífico central con color gris.

\section{ÍNDICE DE EROSIÓN-SEDIMENTACIÓN COSTERA (IE-SC)}

\section{Definición de niveles}

Por medio del IE-SC se clasifica las distintas áreas costeras asignándoles un nivel o peso entre 1 y 5 . El nivel 1 corresponde con áreas sin evidencia de erosión-sedimentación costera (sin afectación o daños evidentes), mientras que el nivel 5 son áreas con severos problemas debido a la pérdida o ganancia de material, erosión exacerbada o sedimentación intensiva. Las evidencias de erosión-sedimentación utilizadas en esta metodología son de tres tipos: a) evidencias en la vegetación, b) evidencias en la infraestructura, y c) evidencias en el medio físico-geológico (materiales inconsolidados). Los parámetros que se utilizan para realizar la evaluación de la costa son, entre otros, formación de terrazas de erosión, exposición de raíces o árboles cercanos a la zona intermareal o bien dentro de ella, presencia de infraestructura que tiene como objetivo la "estabilización" o contención de erosión-sedimentación, etc. Para clasificar un segmento de costa en una u otra categoría se deben observar todas o la mayoría de las características indicadas para cada una de las clases. Luego esta información debe ser codificada en su nivel respectivo utilizando colores o tramas en un mapa temático, el cual debe formar parte de un sistema de información geográfico.

A la hora de utilizar esta metodología se debe tomar en cuenta lo siguiente:

1. El control de campo debe realizarse al menos dos veces al año, tanto en la estación lluviosa como en la estación seca, por un período no menor de dos años. Además, se deben realizar observaciones a lo largo de la línea de costa durante las mareas mensuales más altas y extraordinarias. Evidentemente, cuanto mayor sea el período de observación, mayor es la cantidad de datos y, por ende, la calidad de la información, por lo que tres años es un plazo adecuado. La observación detallada y cartografiado por medio del control de campo debe hacerse tomando en cuenta la morfología de la costa tanto en planta como en perfil. Se han establecido estos tiempos de monitoreo debido a que estos permiten eliminar o al menos minimizar el efecto por cambios estacionales en la costa.

2) Se recomienda construir un registro fotográfico detallado, con el fin de hacer posteriores comparaciones temporales. Los registros fotográficos deben hacer especial énfasis en la zona intermareal.

3) La escala de trabajo puede ser variada pero para obtener datos de calidad que se puedan 
utilizar en la planificación costera se recomiendan escalas de trabajo inferior a 1:25000 sobre todo en áreas de interés. Si a partir de los mapas generales se visualizan áreas críticas, ya sea por la existencia infraestructura civil de importancia (p.ej. puertos, marinas, y poblaciones) o por que se planea desarrollar un área particular, se sugiere la utilización de una escala 1:1000. Para zonas de detalle, se recomiendan mapas con una buena resolución (p.ej. 0,5 m) con el fin de cartografiar detalladamente las zonas de erosión o sitios de sedimentación y asignar los respectivos niveles.

4) Es indispensable el estudio geomorfológico y sedimentológico por medio de fotografías aéreas o satélite, a fin de tener un registro temporalmente más amplio de las variaciones en la costa. A esto se suma, la búsqueda $\mathrm{y}$ análisis de registros antiguos tales como documentos escritos, mapas y fotografías.

5) Son de mucha ayuda las entrevistas a los pobladores del área, a fin de documentar las observaciones, por lo general puntuales, no registradas por medio de los métodos anteriores; además, el registro histórico siempre es importante.

6) Establecer las actividades antrópicas que podrían potenciar los procesos de erosiónsedimentación en la línea de costa y por ende los cambios en la configuración de la costa..

7) Ubicación y mapeo de la infraestructura de estabilización costera. En este punto se debe tomar en cuenta el tipo y disposición de estructura de estabilización y si esta es eficiente o si por el contrario aumenta el proceso de erosión-sedimentación que se quería mitigar.

\section{NIVEL 1: Erosión-sedimentación no apreciable}

Dentro de esta categoría se presentan aquellas zonas costeras, que no presentan ninguno de los criterios que se mencionarán en los otros niveles. Lo cual no quiere decir necesariamente que no se den procesos de erosión-sedimentación solo que su efecto neto es un equilibrio dinámico, que permite que se mantenga la morfología de la costa. (Fig. 2). En estas zonas se debe poner especial atención y monitorear por periodos inclusive más largos, ya que en muchos casos los cambios son muy sutiles.

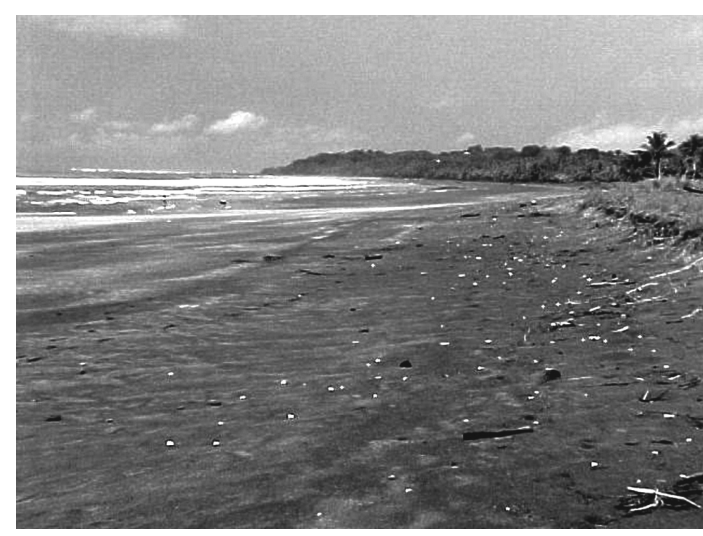

Fig. 2: Playa con índice de erosión-sedimentación costera nivel 1 en la zona de Esterillos.

\section{NIVEL 2: Erosión-sedimentación muy leve}

En este nivel se incluyen:

(1) las zonas costeras donde las raíces de las plantas, como palmeras, árboles y pastos, están ligeramente expuestas en el límite de marea alta normal.

(2) Se observan terrazas de erosión menores a $50 \mathrm{~cm}$ de altura (Fig. 3).

(3) En estas zonas la infraestructura civil no está comprometida, ya sea porque no existe o porque está considerablemente alejada de la zona de marea alta.

\section{NIVEL 3: Erosión-sedimentación media}

(1) Se presenta la exposición de raíces de palmeras y árboles dentro de la zona de marea alta. Las palmeras u otro tipo de vegetación de gran tamaño (mayor a $5 \mathrm{~m}$ ) pueden estar 

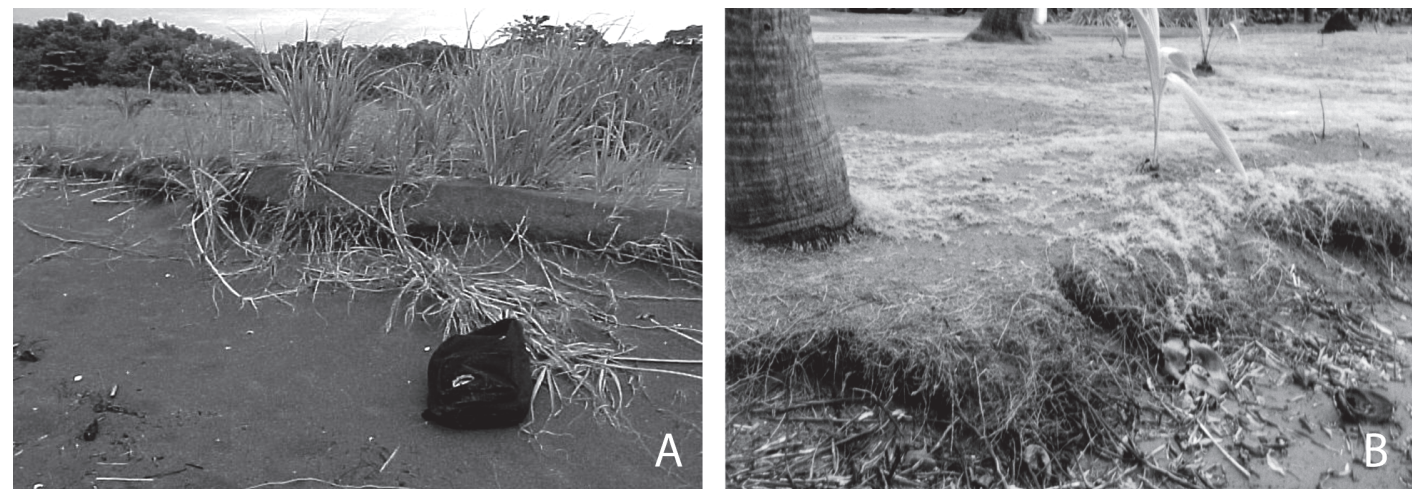

Fig. 3: Zonas costeras nivel 2:

A. Exposición de raíces de pastos de baja densidad (playa Hermosa);

B. Terraza de erosión y leve exposición de raíces (Esterillos Oeste).

basculadas en el límite de las mareas más altas o dentro de la zona intermareal, lo que indica la pérdida del sustrato que las sustenta y su estado ha empezado a ser crítico. Las raíces de las palmeras son cortadas por la acción marina (Fig. 4).

(2) Las terrazas, cuando están presentes, tienen alturas que oscilan entre $50 \mathrm{~cm}$ y $1 \mathrm{~m}$ de altura.

(3) Cuando se presenta infraestructura civil esta se encuentra alejada al menos $5 \mathrm{~m}$ del límite de marea alta.

(4) No se presentan construcciones que tengan como fin la contención de los procesos de erosión-sedimentación costera.

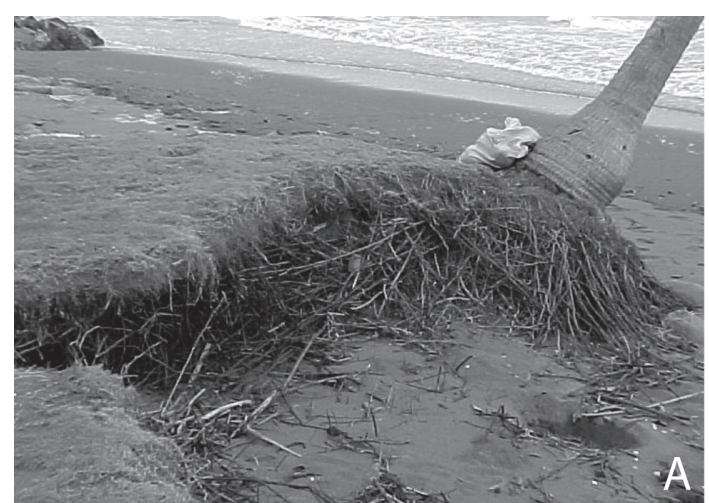

\section{NIVEL 4: Erosión-sedimentación fuerte}

(1) En este nivel el sistema radical de las palmeras y árboles tiene fuerte exposición radicular, en algunos casos las raíces de las palmeras han sido eliminadas y solo queda la parte más cercana al tronco.

(2) La vegetación de gran tamaño (mayor de 5 m) se encuentra tumbada a lo largo de la zona intermareal e incluso es removida por medio del oleaje y las mareas hacia el mar.

(3) Cuando se desarrollan terrazas, estas tienen alturas entre 1 metro a 1,5 metros.

(4) La infraestructura se encuentra cerca del frente de erosión-sedimentación, aproximadamente

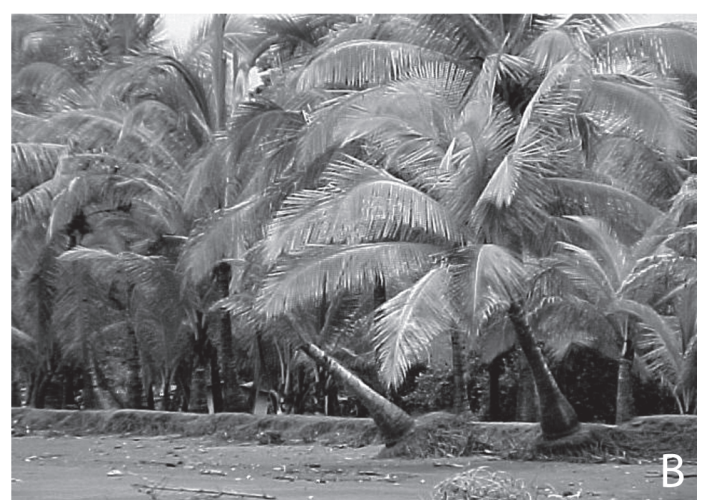

Fig. 4: Zonas costeras nivel 3:

A. Terraza de erosión, raíces expuestas y palmera basculada (playa Esterillos Este); B. Terraza de erosión, raíces expuestas y palmeras basculadas (playa Esterillos Central). 
a dos metros, lo que hace que tenga fuertes posibilidades de ser afectada en el futuro si la relación erosión-sedimentación se mantiene o aumenta.

(5) Debido a la cercanía del frente de erosiónsedimentación, se utilizan sistemas de estabilización costera, como por ejemplo, diques de sacos de arena, muros de roca, chatarra, llantas, muros de cemento, gaviones, etc. Estas construcciones de estabilización son eficientes y si presentan daños, estos son muy leves (Fig. 5).

\section{NIVEL 5: Erosión-sedimentación muy fuerte}

(1) Los sistemas radicales de las palmeras se encuentran rotos, totalmente expuestos $\mathrm{y}$ desarticulados.

(2) El sustrato de las palmeras y árboles adultos (mayor a $5 \mathrm{~m}$ altura) están socavados, tumbados y son removidos por las corrientes.

(3) Si se presentan terrazas de erosión, estas son mayores de 1,5 $\mathrm{m}$ de altura.

(4) Se utilizan los sistemas de estabilización de la costa, los cuales no han sido efectivos, ya que presentan evidentes señales de destrucción, socavamiento, colapso parcial o total y en algunos casos estos son enterrados como parte del mismo proceso de erosión-sedimentación.
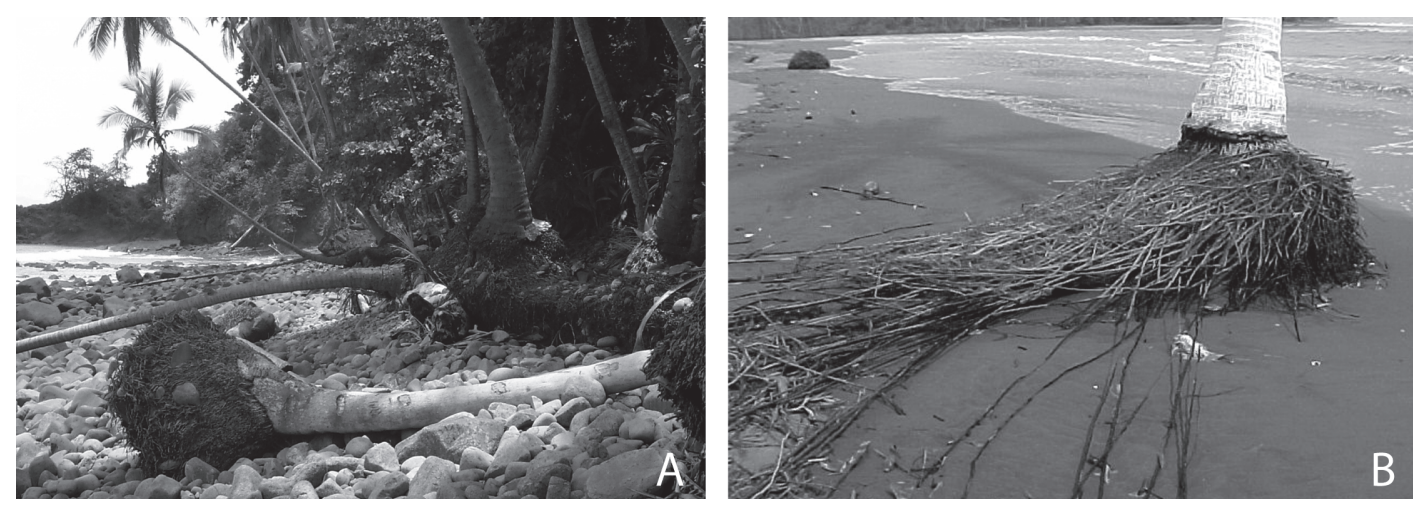

Fig. 5: Zonas costeras nivel 4:

(5) En algunos casos el mal diseño y distribución del sistema de estabilización costera se convierte en un potenciador de la remoción de material, incluso en zonas que no estaban siendo afectadas. Debido a la ineficiencia de la infraestructura de estabilización, la infraestructura civil (casas de veraneo, viviendas permanentes, puertos, atracaderos, etc.) se encuentra comprometida por el oleaje, las mareas, las corrientes litorales o las corrientes fluviales en las desembocaduras, esto provoca la remoción del medio soportante.

(6) Las bases de la infraestructura son falseadas, hay colapso de techos y paredes, llegando incluso a la pérdida total de la infraestructura (Fig. 6).

\section{Presentación de la información}

Los datos generados se muestran utilizando mapas temáticos dentro de un sistema de información que incluyen: a) sitios de erosión intensiva (largo, ancho), asignando los respectivos niveles o pesos con colores o tramas, b) sitios de sedimentación intensiva (largo, ancho), c) cambios morfológicos costeros drásticos, d) mapeo tanto de la infraestructura afectada como de la infraestructura de estabilización costera (sistemas de estabilización), documentando su grado de estabilidad y daños sufridos, si los hubiere. Toda esta información se da en función del tiempo.

A. Palmeras basculadas, tumbadas y en proceso de remoción, terraza de erosión y raíces expuestas (playa Puerto escondido);

B. Palmera en la zona intermareal, la cual ha perdido por erosión el sustrato que la mantenía en pie, raíces completamente expuestas, el sistema radicular destruido (playa Bejuco). 

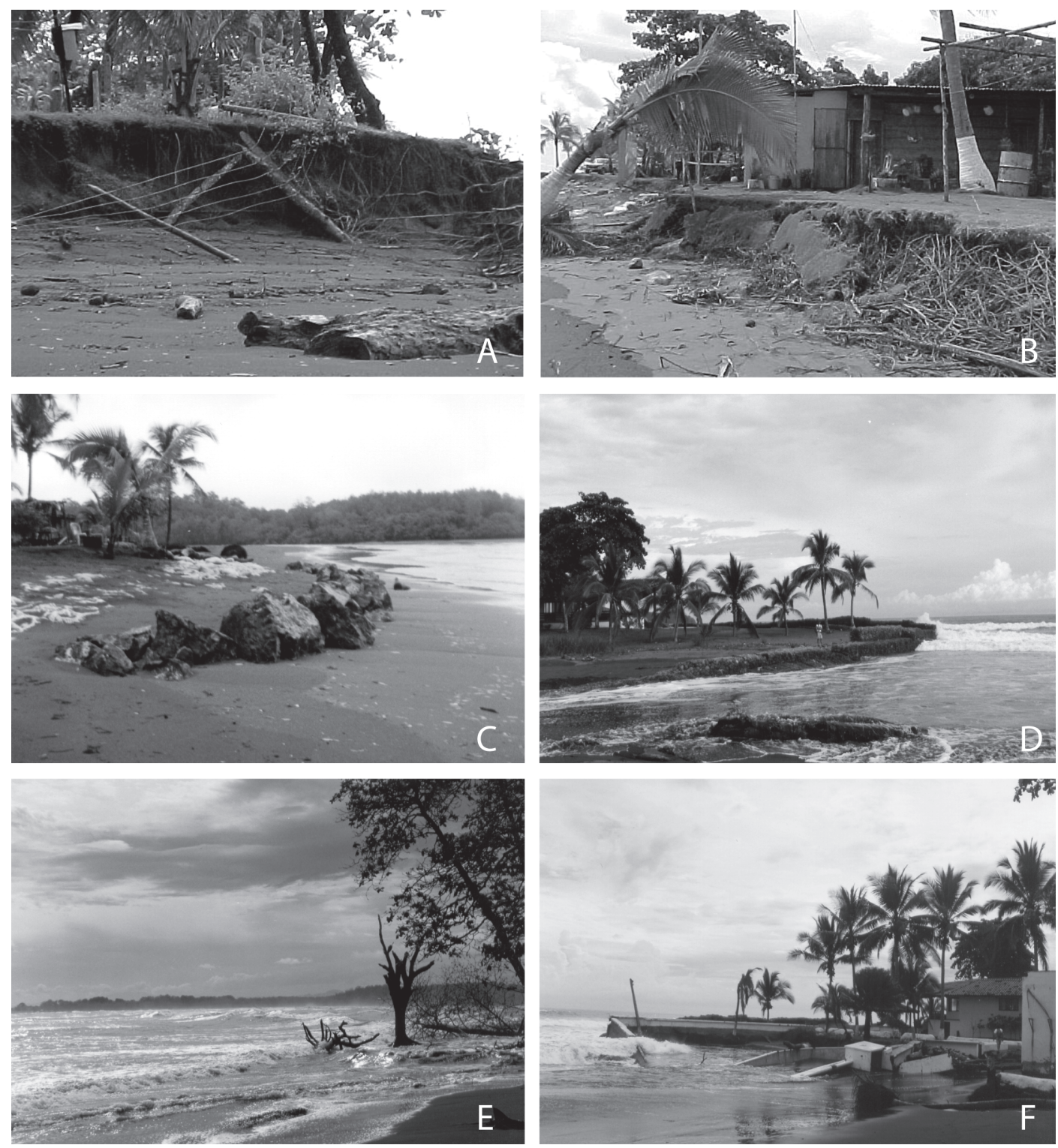

Fig. 6: Zonas costeras nivel 5:

A. Terraza de erosión e infraestructura dañada (playa Esterillos Oeste);

B. Terraza de erosión, raíces expuestas, palmeras basculadas e infraestructura a menos de un metro del frente de erosión (playa Bejuco);

C. Utilización de construcciones para la contención de la erosión: sacos de arena, pequeño muro de roca (playa Bejuco);

D. Utilización de construcciones para la contención de la erosión: gaviones (isla Damas);

E. La presencia de árboles evidencian la erosión y ascenso del nivel de la línea de marea alta (isla Damas);

F. Muro de cemento y gaviones que fueron insuficientes provocando inclusive que se acelerara el proceso de erosión, lo que significó la pérdida total de dos casas de veraneo (isla Damas). 


\section{Ventajas y desventajas del IE-SC}

Como ventajas de esta metodología se pueden citar, que es una herramienta útil en las primeras fases de un estudio integral de manejo costero; por medio de esta se establece el grado de "estabilidad dinámica de las áreas costeras"; por su naturaleza permite la utilización de los sistemas orientados a la toma de decisiones, como ejemplo, planes reguladores; $y$, por último, constituye una herramienta que tiene un costo económico relativamente bajo.

Una desventaja es, que por la naturaleza de los fenómenos estudiados la colección de datos requiere de un tiempo considerable, por lo que el IE-SC no puede emitir criterios sólidos de forma inmediata.

\section{APLICACIONES DE IE-SC}

En la costa del pacífico central de Costa Rica, se presentan zonas constituidas por materiales inconsolidados que son potencialmente vulnerables a los procesos de erosión-sedimentación intensivos ya sea por efectos naturales y/o antrópicos, p.ej., desarrollos urbanísticos y turísticos mal planificados y mal desarrollados, donde no se toman en cuenta la dinámica litoral en el diseño y planificación de las obras. Para aplicar la metodología IE-SC, se ha realizado el estudio de la costa a escala 1:25 000, dicho monitoreo se ha realizado durante lo últimos cuatro años. Se han seleccionado dos áreas para ejemplificar los resultados obtenidos: 1) la desembocadura del río Tárcoles y 2) la zona de Damas cerca de Quepos.

\section{CASO 1: Damas, Quepos}

Los resultados del análisis de la zona de Damas se muestran en la figura 7. En esta se presentan zonas que no muestran efectos de erosiónsedimentación por lo que se les asigna el nivel 1, como es el caso de El Cocal. Sin embargo, la

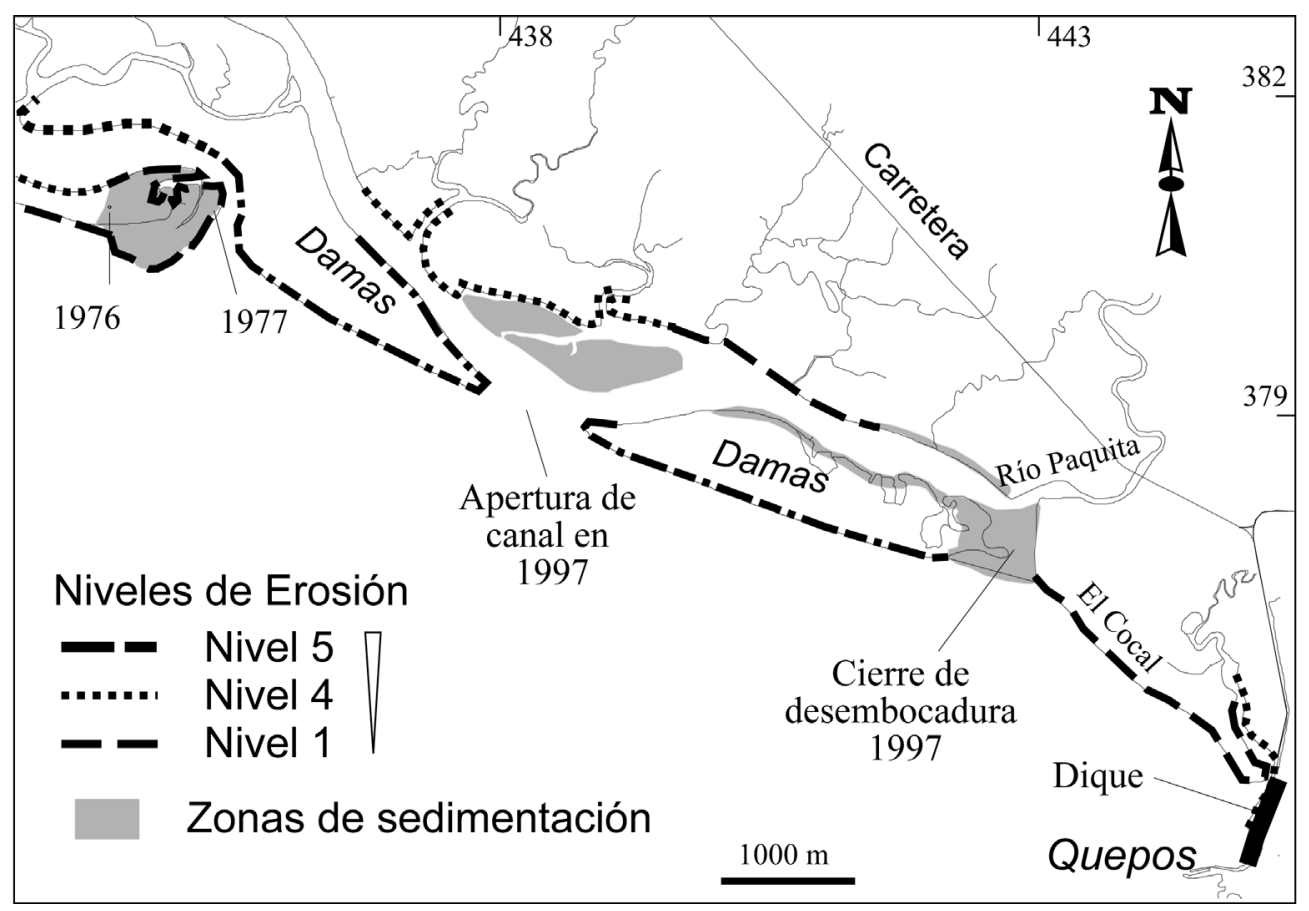

Fig.7: Los niveles de erosión-sedimentación (IE-SC) para el área de Damas-Quepos. 
mayoría del área costera tiene niveles 4 y 5. En estas zonas los procesos de erosión son intensos, ya que remueven el sedimento, principalmente arenoso y lo redepositan en otras zonas de la costa. Un ejemplo de esto son los cambio sufridos en la barra arenosa de Damas donde los sedimentos fueron removidos de la zona central de la barra, partiendo la parte subaérea de la barra en dos, mientras que en la zona de desembocadura del río Paquita se produjo una sedimentación excesiva lo que provocó que el río cambiara su desembocadura hacia el NW; dichos cambios morfológicos se vienen sucediendo desde aproximadamente el año 1997. En esta área las pérdidas económicas han sido cuantiosas, ya que han sido destruidas casas de verano, viviendas permanentes, una pista de aterrizaje, así como cultivos varios.

\section{CASO 2: río Tárcoles}

La zona conocida como playa Guacalillo se cataloga como de nivel 1, pero cerca de desembocadura del río Tárcoles los índices varían entre 4 y 5, lo que implica procesos de erosiónsedimentación intensivos que afectan la infraestructura (Fig.8). En la desembocadura se han perdido una serie de casas tanto de veraneo como de viviendas permanentes, ejemplo de esto se muestra en la figura 9, la casa se construyó en el año 2004. Como se puede ver en la fotografía A, Fig. 9 se construyó un muro de roca para contener el proceso de erosión, pero es evidente en la fotografía B, Fig. 9 que este no fue efectivo para contener la erosión y evitar la pérdida total de la edificación.

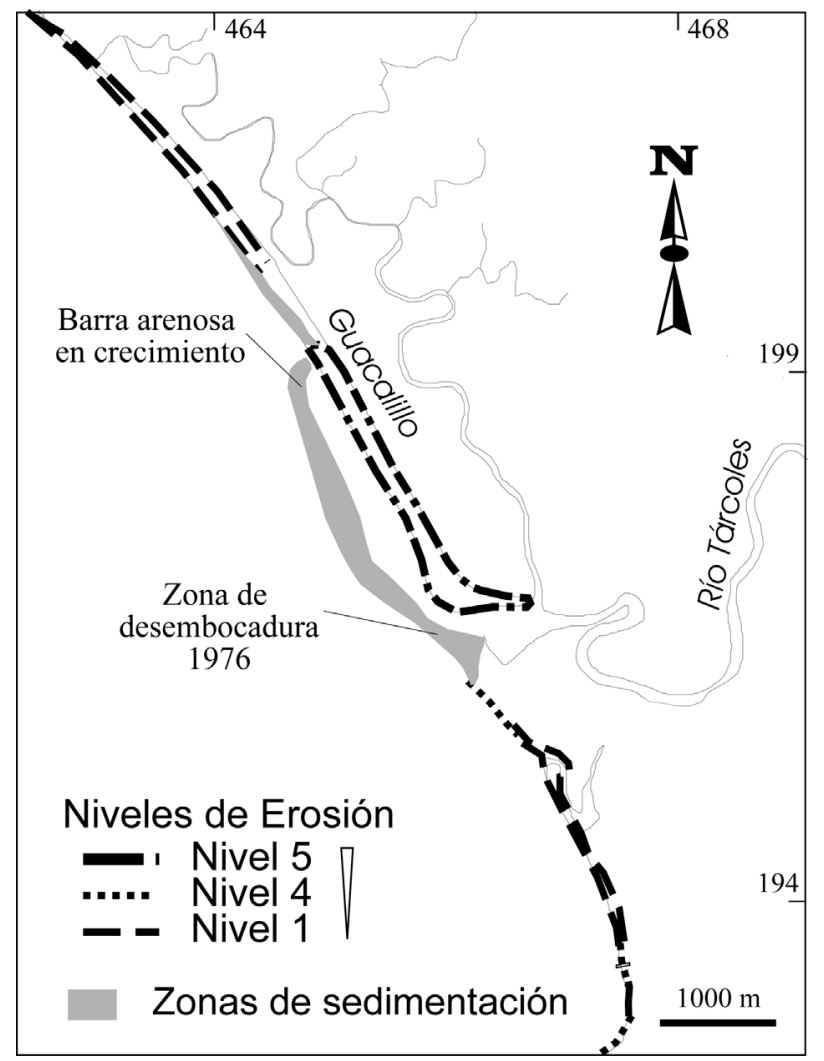

Fig.8: Los niveles de erosión-sedimentación (IE-SC) para el área de la desembocadura del río Tárcoles. 

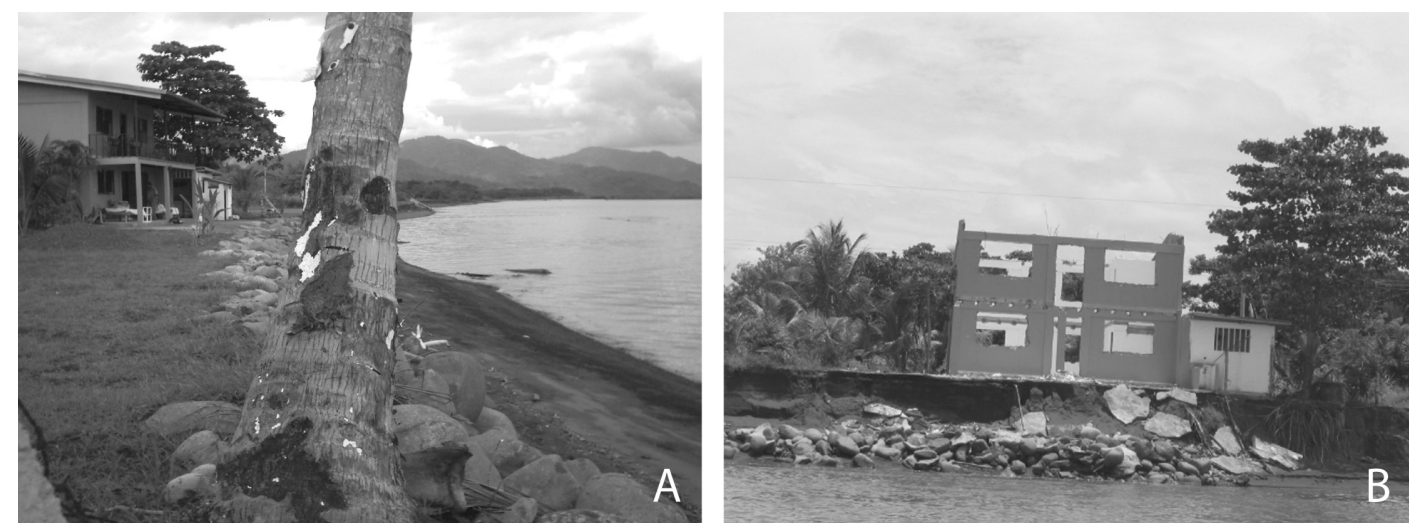

Fig. 9: Zonas costeras nivel 5 en el área del río Tárcoles:

A. Muro de roca creado contener el proceso de erosión (fotografía tomada en julio 2005;

B. Erosión y pérdida total de la edificación (fotografía tomada en agosto 2005).

\section{CONCLUSIONES}

Se ha definido y aplicado por primera vez, la metodología índice de erosión-sedimentación costera (IE-SC), la cual constituye una herramienta de reconocimiento de tipo cualitativo de relativo bajo costo. La herramienta permite establecer el grado de "estabilidad dinámica de las áreas costeras", la cual es deseable conocer para cualquier tipo de actividad que se quiera desarrollar en la zona costera. Esta herramienta debe realizarse en las primeras fases del estudio integral de vulnerabilidad costera.

Se ha determinado que las zonas con mayores problemas de erosión-sedimentación (nivel 5) son las zonas frontales de los sistemas estuarinos, así como las barras arenosas ya que estas son las zonas costeras donde el oleaje y las corrientes litorales actúan de manera más eficiente en la erosión, transporte y sedimentación de materiales. En el caso de los sistemas estuarinos los materiales acarreados son limos, arcillas $\mathrm{y}$ arenas finas, mientras que en las playas son arenas medias. Los procesos de erosión-sedimentación se intensifican durante las mareas más altas mensuales y anuales. Particularmente en la zona del pacífico central costarricense se determinaron dos áreas donde el índice de erosiónsedimentación tiene elevados valores (niveles 4 y 5), a saber, en la zona de Damas y en la zona de la desembocadura del río Tárcoles. Ambas zonas se caracterizan por presentar procesos erosivos intensivos, con pérdida de infraestructura. Los procesos de sedimentación también son intensivos y constituyen, en su gran mayoría, la resedimentación de los materiales erosionados en las zonas aledañas.

\section{AGRADECIMIENTOS}

Agradecemos a la Viccrrectoría de Investigación (UCR) por el financiamiento otorgado. Asimismo a los compañeros Pedro Acosta, Enrique Quintanilla y Carlos Vargas por su valioso apoyo en el trabajo de campo, en la Escuela Centroamericana de Geología a Marco Barahona y Percy Denyer. A la geóloga Johanna Méndez de la Sección de prevención de la Comisión nacional de prevención de riesgo y atención de amenazas (CNE). Este es un aporte a los proyectos de investigación \# 113-A2-527 y \# 113-A4-071 de la Universidad de Costa Rica

\section{REFERENCIAS}

Anderson, J., Rodríguez, A., Flecher, C., \& Fitzgerald, D., 2001: Researches focus attention on coastal response to climate changes. - EOS, 82(44): 513, 519-520. 
Cárdenes, G., 2003: Evolución de los sistemas sedimentarios costero y aluvial de la región de Parrita, pacífico central de Costa Rica. - Rev. Geol. Amér. Central, 28: 69-76.

Gornitz, V., Beaty, T. \& Daniels, R., 1997: A coastal hazards data base for the US west coast. - 162 págs. ORNLICDIAC-81, NDP-043C. Oak Ridge National Laboratory. Oak Ridge, Tennessee.

Lizano, O. 1997. Las mareas extraordinarias de 1997 en la costa del Pacífico de Costa Rica. - Tóp. Meteor. Oceanogr. 4: 169-179.

Nichols, R.,1998: Coastal vulnerability assessment for sealevel rise: Evaluation and selection of methodologies for implementation. - 44 págs. Caribbean Planning for Adaptation to Global Climate Change (CPACC) Project Component 6: Coastal Vulnerability and Risk Assessment. Technical Report TR 98002. UN.

Ortiz, P., 1994: Repercusiones del ascenso del mar en costas de planicies deltaicas. - En: Geografía y Desarrollo, 11(2): 69-72. México.
Sharples, C,. 2004: Indicative mapping of Tasmanian coastal vulnerability to climate change and sea level rise. Explanatory Report. - 130 págs. Dep. Primary Industries, Water \& Environment (Tasmania). Tasmania, Australia.

Thieler, E., Hammar-Klase, E., Hole, W., 1999: National assessment of coastal vulnerability to sea-level rise: Preliminary results for the US Atlantic Coast. US Geological Survey. Open-file Report 99-593. (On line file: HTTPS://pubs.usgs.gov/of99593/index.html).

Vafeidisa, A.T., Nicholls, R.J., McFaddena, L., Hinkelc, J. \& Grashoffd, P.S., 2004: Developing a global database for coastal vulnerability analysis: Design issues and challenges. - Internat. Archives Photogrammetry, Remote Sensing and Spatial Inf. Sci. 34, Part XXX: 5. 